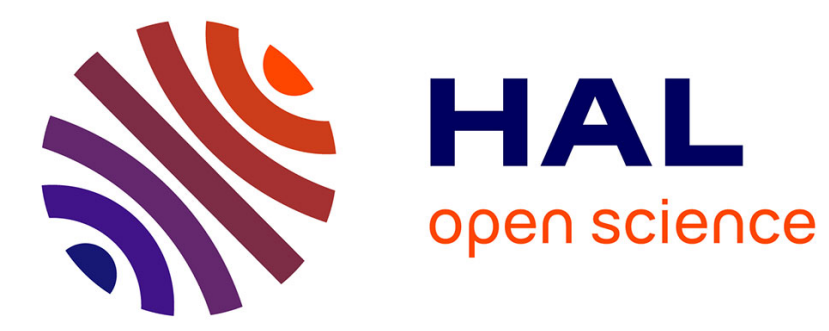

\title{
Autoportrait et emblématique princière à la fin du Moyen Âge
}

Laurent Hablot

\section{To cite this version:}

Laurent Hablot. Autoportrait et emblématique princière à la fin du Moyen Âge. Le Moyen Age. Revue d'histoire et de philologie, 2016, Autoportrait et représentation de l'individu, 122 (1), pp.67-81. 10.3917/rma.221.0067 . halshs-02614828

\section{HAL Id: halshs-02614828 https://shs.hal.science/halshs-02614828}

Submitted on 25 May 2020

HAL is a multi-disciplinary open access archive for the deposit and dissemination of scientific research documents, whether they are published or not. The documents may come from teaching and research institutions in France or abroad, or from public or private research centers.
L'archive ouverte pluridisciplinaire HAL, est destinée au dépôt et à la diffusion de documents scientifiques de niveau recherche, publiés ou non, émanant des établissements d'enseignement et de recherche français ou étrangers, des laboratoires publics ou privés. 


\title{
AUTOPORTRAIT ET EMBLÉMATIQUE PRINCIÈRE À LA FIN DU MOYEN ÂGE
}

\author{
Laurent Hablot
}

De Boeck Supérieur | «e Moyen Age »

2016/1 Tome CXXII | pages 67 à 81

ISSN 0027-2841

ISBN 9782807390676

Article disponible en ligne à l'adresse :

https://www.cairn.info/revue-le-moyen-age-2016-1-page-67.htm

Distribution électronique Cairn.info pour De Boeck Supérieur.

(C) De Boeck Supérieur. Tous droits réservés pour tous pays.

La reproduction ou représentation de cet article, notamment par photocopie, n'est autorisée que dans les limites des conditions générales d'utilisation du site ou, le cas échéant, des conditions générales de la licence souscrite par votre établissement. Toute autre reproduction ou représentation, en tout ou partie, sous quelque forme et de quelque manière que ce soit, est interdite sauf accord préalable et écrit de l'éditeur, en dehors des cas prévus par la législation en vigueur en France. Il est précisé que son stockage dans une base de données est également interdit. 


\section{Autoportrait et emblématique princière à la fin du Moyen Âge}

Dans toutes civilisations, la vocation même de l'emblème est de représenter une personne physique ou morale au moyen d'un signe ${ }^{1}$. Celui-ci produit donc une représentation par l'image de cette personne, un portrait au premier sens du terme ${ }^{2}$. Comme toute société, le Moyen Âge occidental a généré différents systèmes de signes chargés de faire connaître et reconnaître les gens et les choses. Si l'on sait peu de choses de l'emblématique du haut Moyen Âge, la réorganisation sociale et religieuse du $\mathrm{xII}^{\mathrm{e}}$ siècle, développée en lien avec la féodalité, a contribué à la naissance d'un système tout à fait original et performant qui existe encore aujourd'hui : l'héraldique. Concentrés sur un cadre - généralement, mais pas exclusivement, en forme de bouclier -, ces signes, les armoiries, servent prioritairement à désigner un individu et s'élaborent selon un ensemble de règles, le Blason, qui en gère les formes, les couleurs, les compositions, le style et les pratiques. Ce système, très efficace mais également très contraignant, s'impose rapidement à tout l'Occident sans restriction ni de condition ni de genre ${ }^{3}$. Cette généralisation n'empêche pourtant pas toute créativité dans ce domaine. Aux côtés des armoiries apparaissent bientôt d'autres signes emblématiques destinés à distinguer les personnes. Ce sont, au début du XIII ${ }^{\mathrm{e}}$ siècle, les cimiers - décor des casques -, les supports - animaux ou objets soutenants l'écu -, les cris de guerre ${ }^{4}$ et bientôt, au milieu du XIV siècle, les devises, signes librement

1. En ce sens, on le distingue du symbole chargé de donner corps imagé à une idée, à un concept.

2. J.C. Sснмітт revient sur le sens de ce mot dans La mort, les morts et le portrait, Le Portrait individuel. Réflexions autour d'une forme de représentation XIII-XVe siècles, éd. D. OLARIU, Berne-Berlin-Bruxelles-FrancfortNew York-Oxford-Vienne, 2009, p. 17-19.

3. Sur ces questions, voir M. Pastoureau, Traité d'héraldique, $5^{\mathrm{e}}$ éd., Paris, 2008. Pour une discussion sur les fonctions sociales de l'héraldique au Moyen Âge voir aussi mon article Les armoiries, un marqueur du rang dans les sociétés médiévales ?, Rank and Order. The Formation of Aristocratic Elites in Western and Central Europe, 500-1500, éd. J.H. Peltzer, Ostfildern, 2015, p. 245-270.

4. Sur le sujet, qui ne sera pas développé ici, voir mon article Les cris écrits. L'apparition des cris, mots emblématiques et sentences dans l'héraldique médiévale, un regard sur la voix et l'écrit, La voix et l'écrit, Pris-ma, t. 23/45-46, 2007, p. 22-52. 
figurés associés à une sentence, le mot, à des couleurs et des lettres ${ }^{5}$. À la fin de la période, l'apparition du portrait réaliste, emblème ultime, solde en quelque sorte ces tentatives successives de désignation de l'individu ${ }^{6}$.

La naissance du portrait réaliste entretient en effet des liens très étroits avec l'emblème. M. Pastoureau a déjà souligné la proximité sémiologique et graphique entre ces formes de représentations, idée reprise dans de nombreux travaux et analyses de portraits médiévaux ${ }^{7}$. Le choix fréquent, à l'origine, de la figuration de profil et l'insistance sur certaines caractéristiques physiques, proprement "emblématiques " comme un nez busqué ou un prognathisme héréditaire ${ }^{8}$, apparentent le visage aux animaux stylisés des armoiries, graphiquement construits sur les mêmes principes d'exagération des spécificités morphologiques. Mais ce qui confirme plus que tout la proximité sémiologique de ces signes est leur très fréquente association dans les outils de représentation du Moyen Âge finissant. Il existe ainsi de nombreux exemples d'objets qui assemblent sur un même support - médaille, mais aussi enluminures, vitraux, bijoux, monnaies, sceaux, peintures de chevalet, gisants - les différentes formes de «portrait» du commanditaire : visage réaliste, couleurs, armoiries, cimier, devise, mot, chiffre, etc. C'est le cas par exemple du célèbre portrait de Francesco d'Este peint par Rogier Van der Weyden vers $1460^{\circ}$. L'ensemble de ces outils de reconnaissance interfère alors avec les autres signes de l'identité comme la signature, le nom ou le sceau ${ }^{10}$. Ils répondent tous, au moment de leur assomption, à des besoins précis liés aux évolutions de la société qui les génère. Mais, comme toutes réalités médiévales, ces outils ne s'effacent jamais à mesure que d'autres s'inventent.

5. Sur le sujet voir ma thèse à paraître chez Brepols, La Devise, mise en signe du prince, mise en scène $d u$ pouvoir et, pour une synthèse, voir ID., La devise, un nouvel emblème pour les princes du Xve siècle, $L a$ création artistique en France autour de 1400. Actes du colloque international École du Louvre-Musée des Beaux-Arts de Dijon-Université de Bourgogne, École du Louvre, 7 et 8 juillet 2004, Musée des Beaux-Arts de Dijon-Université de Bourgogne, 9 et 10 juillet 2004, éd. É. Taburet-Delahaye, Paris, 2006, p. 177-192.

6. La bibliographie sur cette question est abondante. Pour une synthèse récente voir par exemple $L e$ portrait individuel.

7. M. Pastoureau, L'effervescence emblématique et les origines héraldiques du portrait au XIV siècle, Bulletin de la Société nationale des Antiquaires de France, 1985 (1987), p. 108-115 ; C. DE MÉRINDOL, Portrait et généalogie. La genèse du portrait réaliste et individualiste, $118^{e}$ Congrès national des sociétés historiques et scientifiques. Histoire médiévale. Population et démographie, Pau, 1993, p. 219-248 ; J.B. DE VAIVRE, Sur trois primitifs français du $x^{e}$ siècle et le portrait de Jean le Bon, Gazette des Beaux-arts, avr. 1981, p. 131-156 ; ID., Représentations de Louis $\mathrm{I}^{\mathrm{er}} \mathrm{d}^{\prime}$ Anjou et portraits de Louis II, Comptes rendus des Séances de l'Académie des Inscriptions et Belles-Lettres, t. 128, 1984, p. 722-745. En dernier lieu voir la réflexion fondamentale de H. Belting, Wappen und Porträt. Zwei Medien des Körpers, Bild-Anthropologie. Entwürfe für eine Bildwissenschaft, Munich, 2001, p. 115-142 et sur cette question et celle, plus large, des relations entre portrait/ armoires et devises, voir S. SLANISKA, La fonction distinctive des ordres et du portrait noble dans les sociétés de cour (XIV ${ }^{\mathrm{e}}-\mathrm{XVII}^{\mathrm{e}}$ siècles), Signes et couleurs des identités politiques du Moyen Âge à nos jours, éd. M. AurELL, D. Turel, C. Manigand, J. Grévy, L. Hablot, C. Girbea, Rennes, 2008, p. 313-332.

8. S. Perkinson, The Likeness of the King. A Prehistory of Portraiture in Late Medieval France, Chicago, 2009 ; C.R. Sherman, The Portraits of Charles V of France, Zeitschrift für Kunstgeschichte, t. 34, 1971, p. $72-88$.

9. New York, Metropolitan Museum of Art, Inv. 32.100.43.

10. Pour l'étude des relations entre héraldique et onomastique, voir M. NAssiet, Nom et blason. Un discours de la filiation et de l'alliance ( IIV $^{\mathrm{e}}-\mathrm{XVIII}^{\mathrm{e}} \mathrm{s}$.), L'Homme, t. 34/129, 1994, p. 5-30. Pour une synthèse sur le nom au Moyen Âge, voir en dernier lieu M. Bourin, P. Chareille, Noms, surnoms et prénoms au Moyen Âge, Paris, 2014 ; pour les fonctions emblématiques de la signature, voir B. FrAENKEL, La Signature. Genèse d'un signe, Paris, 1992 ; C. JEAy, Signature et pouvoir au Moyen Âge, Paris, 2015. 
Ils se superposent au contraire pour parfaire la représentation de celui dont ils « tirent » les traits. Les médailles du Quattrocento, véritables miroirs à facettes de l'individu qui cumulent ces différentes formes de représentation, en témoignent ${ }^{11}$.

Le lien entre l'emblème et le portrait est donc évident ${ }^{12}$. L'emblème n'est finalement qu'un portrait parmi d'autres, inscrit dans un registre de signes ni plus ni moins contraignant, ni plus ni moins riche de sens et de pertinence que le portrait réaliste. Reste pourtant à savoir de quoi nous parlons exactement. Qu'est-ce qu'un portrait pour un homme du Moyen Âge ? D'après E. Auerbach, il s'agit alors d'une " conception figurative de la réalitée ${ }^{13}$ », dont les historiens soulignent, à l'instar de J.C. Schmitt ou de B. Bedos-Rezak, qu'elle inscrit dans le "plan divin » une réalité extérieure élevée à un sens supérieur. Il s'agira alors plutôt de l'« image » d'un individu dont les traits ne sont pas encore individualisés, investie de finalités sociales et eschatologiques. Certaines d'entre elles sont même produites sous le contrôle immédiat de leur modèle, sorte $\mathrm{d}^{\prime}$ « auto-images » dans lesquelles la finalité religieuse - l'accès individuel au Salut - entre en tension avec la finalité sociale - la définition d'une condition dans un stéréotype ${ }^{14}$.

Existe-t-il pour autant des autoportraits emblématiques ${ }^{15}$ ? L'emblème peut-il servir cette "expression la plus radicale de la relation de sujet à sujet $^{16}{ }^{\star}$ ? Quel contrôle le détenteur de l'emblème exercice-t-il sur la composition de ce dernier. L'a t-il choisi ? L'a-t-il créé ? L'a-t-il représenté ? Assurément quelques exceptions permettent de deviner ou de reconnaître derrière le promoteur de l'emblème l'utilisateur lui-même. Pourtant, la plupart du temps cette information nous échappe. Un grand nombre de ces emblèmes ne peuvent d'ailleurs pas, du fait même de leurs structures, être considérés comme des autoportraits, c'est notamment le cas des armoiries héritées - donc de la majeure partie d'entre elles en réalité - sur lesquels l'utilisateur n'intervient presque en aucune façon.

En dépit de ces réserves, chaque système de signe est pourtant bien pensé, à l'origine, comme un outil d'autoreprésentation et peut à ce titre générer des « autoportraits emblématiques ». À la fin du Moyen Âge, un individu

11. Voir notamment M. PAstoureau, La naissance de la médaille. Le phénomène emblématique, Revue de Numismatique, 6e sér., t. 24, 1982, p. 206-221 ; ID., Naissance d'une image nouvelle. La médaille du Quattrocento, une emblématique nouvelle, Couleurs, Images, Symboles, Paris, 1989 ; R. Rugolo, Les médailles, Pisanello, éd. L. Puppi, Paris, 1996, p. 138 s.

12. Voir par exemple, pour une application précise et parfaitement conduite, l'étude de L. VISSIÈRE, Les signes et le visage. Etude sur les représentations de Louis II de La Trémoille, Journal des Savants, 2009/2, p. 211-282.

13. Mimésis. La Représentation de la réalité dans la littérature occidentale, Berne, 1946, p. 549-553, cité par Sснмітт, La mort, les morts et le portrait.

14. Ibid., p. 21-23.

15. Suivant les travaux réunis dans le riche volume collectif L'Individu au Moyen Âge. Individuation et individualisation avant la modernité, éd. B.M. Bedos-RezAK, D. IognA-Prat, Paris, 2005, il est possible de distinguer individuation = utilisation de signes d'identification normatifs, et individualisation = expression de l'autoréflexion, de l'écriture de soi.

16. Sснмiтt, La mort, les morts et le portrait, p. 32. 
« se dit » par tous les moyens à sa disposition. Pourtant, à y regarder de plus près, tous ces registres de signes ne portent pas le même reflet de la personne. Il convient donc, pour en saisir les nuances et tenter de déterminer dans quelle mesure ils peuvent être des autoportraits, de revenir sur ces différents systèmes sémiologiques et leur relation ontologique à leur utilisateur.

\section{Les armoiries, un autoportrait?}

La plupart des auteurs s'accordent pour expliquer l'origine des armoiries par la nécessité faite aux chevaliers dissimulés sous leur haubert et leur casque à nasal de se trouver des signes de reconnaissance capables, dans les combats, de se substituer à leur visage ${ }^{17}$. L'épisode de Guillaume le Conquérant contraint de relever son casque à Hastings en 1066 pour se faire reconnaître et redonner confiance à son armée qui le croyait mort est fréquemment invoqué comme terminus a quo du processus. Même si cette lecture de la genèse de l'héraldique est aujourd'hui contestée ${ }^{18}$, il n'en reste pas moins qu'elle énoncel'idée quel'armoirie constitue un autre visage. Estce réellement le cas ? Les pratiques héraldiques postérieures semblent bien le confirmer. Véritable projection emblématique de son titulaire, l'armoirie devient un substitut de la personne qui la rend présente là où elle n'est pas visible, qu'elle soit dissimulée sous son armure ou réellement absente.

17. M. Pastoureau, L'origine militaire des armoiries, Archéologie militaires. Les pays du Nord, Actes du 101 Congrès national des sociétés savantes. Section d'archéologie et d'histoire de l'art, Paris, 1978, p. 107-118. Et ID., Traité d'héraldique, p. 27 : «il est aujourd'hui définitivement admis que cette apparition (des armoiries) n'est en rien due aux croisades, ni à l'Orient, ni à l'Antiquité gréco-romaine, ni même aux envahisseurs germains, mais qu'elle est simplement liée à l'évolution de l'équipement militaire entre la fin du $\mathrm{XI}^{\mathrm{e}}$ siècle et le milieu du XII ${ }^{\mathrm{e}}$ siècle »; Ibid., p. 300 : «Il est définitivement établi que la cause matérielle - mais non unique, je l'ai dit - qui joue un rôle de catalyseur sur l'apparition des premières armoiries, est essentiellement militaire et liée à l'évolution de l'équipement défensif des combattants entre la fin du XI ${ }^{\mathrm{e}}$ siècle et le milieu du XII ${ }^{\mathrm{e}}$ siècle. Rendus à peu près méconnaissables [...] ces combattants ont progressivement (l'adverbe est important) pris l'habitude de faire peindre sur la grande surface plane de leur bouclier en amande des figures-géométriques, animales, florales - leur servant de signes de reconnaissance au cœur de la mêlée [...] ». L'auteur est depuis revenu sur ces considérations en soulignant le rôle prépondérant du tournoi en la matière (ID., L'Art héraldique au Moyen Âge, Paris, 2009, p. 26).

18. La fonctionnalité proprement militaire des armoiries est finalement peu documentée avant la production, au XIII ${ }^{\mathrm{e}}$ siècle, d'images de chevaliers armoriés, davantage tournoyeurs que soldats. Les finalités sémiologiques de ces signes sont avant tout sociales et politiques, ce qui autorise leur très rapide diffusion hors du groupe des guerriers. Voir mon article Entre pratique militaire et symbolique du pouvoir, l'écu armorié au XII ${ }^{e}$ siècle, Estudos de Heráldica medieval, éd. M. Metelo de Seixas, M.d.L. Rosa, Lisbonne, 2012, p. 143-165. A. AILES semble être un des premiers à avoir proposé une analyse critique de la question, il y a maintenant plus de dix ans, dans son article essentiel The Knight, Heraldry and Armour. The Role of Recognition and the Origins of Heraldry, Medieval Knightood IV. Papers from the fifth Strawberry Hill Conference, éd. C. HARPERBill, R. HARveY, Woodbridge, 1990, p. 1-21. R. JonEs a prolongé le débat dans son article Identifying the Warrior on the Pre-Heraldic Battlefield, Anglo-Norman Studies. xxx. Proceedings of the Battle Conference 2007, éd. C.P. LewIs, Woodbridge, 2008 et, plus récemment encore, dans son ouvrage Bloodied Banners. Martial Display on the Medieval Battlefield, Woodbridge, 2011. Un certain nombre des questions posées en 1990 par A. Ailes ont été partiellement résolues par l'auteur dans une communication présentée, en novembre 2011, à l'occasion du colloque international tenu à Poitiers : Heraldry as Markers of Identity in the Medieval Literature. Fact or Fiction?, Marqueurs d'identité dans la littérature médiévale. Mettre en signe l'individu et la famille (XII ${ }^{e}-\mathrm{XV}^{e}$ s.). Actes du colloque tenu à Poitiers les 17 et 18 novembre 2011, éd. C. Girbea, L. Нablot, R. Radulescu, Turnhout, 2014, p. 181- 191. 
L'association très rapide de ces signes héraldiques aux sceaux, eux-mêmes déjà chargés $\mathrm{d}^{\prime}$ une très forte capacité $\mathrm{d}^{\prime}$ abstraction ${ }^{19}$, a sans aucun doute très largement contribué à cette capacité présentielle des armoiries. La mise en scène de ces signes, les règles et stratégies de l'exposition héraldique, les principes de la latéralisation du blason ${ }^{20}$, vont tous dans ce même sens. L'écu armorié existe à la place de celui qui l'utilise. À ce titre, il peut signifier la présence virtuelle de son titulaire, être couronné comme la tête même du prince $^{21}$, honoré par un dais, porter un collier d'ordre de chevalerie ${ }^{22}$, être vêtu de deuil ${ }^{23}$, être jugé et diffamé par contumace ${ }^{24}$, associé au reste de la panoplie militaire. H. Belting souligne d'ailleurs que dans les langues germaniques, l'allemand et le néerlandais, le même terme schild désigne l'écu et le portrait réaliste pour lesquels, dans la Flandre des $\mathrm{XIV}^{\mathrm{e}}$ et $\mathrm{XV}^{\mathrm{e}}$ siècles, officiaient alors les mêmes artistes, les schilderer ${ }^{25}$. Dans les sources françaises du temps, c'est le même terme tableau - table de bois - qui est employé pour désigner à la fois les panneaux héraldiques et les portraits. Ces peintres héraldistes, parmi lesquels on compte nombre des premiers " portraitistes » comme Jean Malouel, Colard de Laon, Jan van Eyck, Pisanello, Barthelemy d'Eyck, Jean Fouquet, auraient ainsi, de façon expérimentale, employé le revers du support usuel des panneaux armoriés - destinés à être exposés en

19. À ce sujet, voir B. Bedos-ReZAK : Signes d'identité et principes d'altérité au XII ${ }^{\mathrm{e}}$ siècle. L'individu, c'est l'autre, L'Individu au Moyen Âge, p. 43-57 ; ID., Medieval Identity. A Sign and a Concept, American Historical Review, t. 105, 2000, p. 1489-1533; ID., When Ego was Imago. Signs of Identity in the Middle Ages, Leyde, 2010 ; ID., L'au-delà du soi. Métamorphoses sigillaires en Europe médiévale, Cahiers de Civilisation médiévale, t. 49, 2006, p. 337-358 ;ID., Une image ontologique : sceau et ressemblance en France préscolastique (1000-1200), Études d'histoire de l'art offertes à Jacques Thirion, éd. A. ErLANDE-Brandenburg, J.M. Leniaud, Paris, 2001, p. 39-50 ; ID., Le sceau et l'art de penser au xiI ${ }^{\mathrm{e}}$ siècle, Pourquoi les sceaux ? La sigillographie, nouvel enjeu de l'histoire de l'art, éd. M. GIL, J.L. Chassel, Villeneuve d'Ascq, 2011, p. 153-176.

20. La description des armoiries, le blasonnement, considère le point de vue du porteur de l'écu et non celui du lecteur. On commencera donc toujours à décrire ces armes par le coin supérieur gauche : la dextre en héraldique, en réalité la droite du chevalier virtuel présent derrière son écu. Sur le sujet, voir H. BeLTiNG, Le portrait médiéval et le portrait autonome. Une question, Le Portrait individuel, p. 123-136, notamment p. 128-129 et L. Навцот, Aux origines de la dextre héraldique. Écu armorié et latéralisation au Moyen Âge, Cahiers de Civilisation médiévale, t. 56, 2013, p. 281-294 ; L'armoirie et les sens au Moyen Âge, Les cinq sens, éd. É. Palazzo, Paris, 2016, p. 565-588.

21. L'apparition conjointe du concept politique de la couronne et de cet attribut sur les armoiries des souverains ne semble pas avoir retenu l'attention des spécialistes des idées politiques. On sait pourtant l'enjeu symbolique et politique que ces figurations de couronne portaient dans la France de la fin du Moyen Âge, notamment dans les conflits entre la monarchie française et les principautés angevine ou bretonne. Sur le sujet, voir mon article Caput regis caput regni. L'image de la tête royale dans le discours politique aux $\mathrm{XII}^{\mathrm{e}}-$ XIII ${ }^{\mathrm{e}}$ siècles, Comunicazione e propaganda nei secoli XII X XIII. Atti del convegno internazionale (Messina, 24-26 maggio 2007), éd. R. Castano, F. Latella, T. Sorrenti, Rome, 2007, p. 349-357 ; M. Jones, En son habit royal. Le duc de Bretagne et son image vers la fin du Moyen Âge, Représentation, pouvoir et royauté à la fin du Moyen Âge, éd. J. Blanchard, Paris, 1995, p. 253-278.

22. Sur cette question et celle plus large des relations entre portrait/armoires et devises, voir SLANISKA, La fonction distinctive des ordres et du portrait noble.

23. C'est le cas par exemple dans les chapelles d'ordres de chevalerie pour les chevaliers décédés. Cet usage annonce celui de la litre funéraire.

24. Voir à ce sujet mon article Sens dessoubz dessus. Le blason de la trahison au Moyen Âge, La Trahison au Moyen Âge. De la monstruosité au crime politique ( $V^{e}-X V^{e}$ siècle), éd. M. BILloré, M. SorIA, Rennes, 2009, p. 331-347.

25. Sur le sujet voir Belting, Le portrait médiéval et le portrait autonome, notamment p. 128-129. Voir aussi du même Wappen und Porträt. Zwei Medien des Körpers, Das Porträt vor der Erfindung des Porträts, éd. M. BüCHSEL, P. SCHMIdT, Mayence, 2003, p. 89-100. 
lieu et place de leur titulaire dans les espaces de pouvoir et de mémoire pour y faire figurer le portrait réaliste du commanditaire. Comme si le chevalier, depuis toujours virtuellement présent derrière l'écu, prenait enfin corps de l'autre côté de la planche.

Suivant la conception médiévale du portrait énoncée plus haut, à la fin du Moyen Âge, l'armoirie est clairement le signe au moyen duquel les élites se font reconnaître de Dieu au jour du Jugement, ce qui reste de soi quand le portrait de chair a disparu. Sous-entendue depuis les origines de l'héraldique $^{26}$, cette fonction du signe est rendue évidente dans l'iconographie funéraire de la fin du Moyen Âge qui expose les armoiries et le portrait aux côtés du défunt ${ }^{27}$. Cette idée se retrouve par exemple dans le thème du "roi mort » cher à René d'Anjou ${ }^{28}$ ou dans celui de l'ange écuyer, véritable transposition médiévale de l'imago clipeata du Bas-Empire qui conduisait devant les dieux le portrait réaliste du défunt supporté par deux génies ailés ${ }^{29}$. Au milieu du $x^{\mathrm{e}}$ siècle, les sculpteurs italiens comme Donatello s'emparent du motif antique et remplacent le portrait du mort par un tondo à ses armes tenu par deux anges ou deux putti.

L'écu armorié peut-il pour autant être considéré comme un portrait directement produit par celui qu'il représente ? Quelle est la latitude d'intervention de l'utilisateur dans la composition et la réalisation de ses armoiries?

Le processus initial de choix des compositions héraldiques médiévales est mal connu. À l'exception de quelques cas documentés où l'on est renseigné sur les motivations d'adoption de telle ou telle figure ou de telles couleurs ${ }^{30}$,

26. Voir à ce sujet mon article L'héraldisation du sacré aux $\mathrm{XII}^{\mathrm{e}}-\mathrm{XIII}^{\mathrm{e}}$ siècles. Une mise en scène de la religion chevaleresque?, Chevalerie et christianisme aux XII et XIII siècles, éd. M. Aurell, C. GIRBEA, Rennes, 2011, p. 211-233.

27. Il existe de nombreux témoignages, pour la fin $\mathrm{du} \mathrm{xv}^{\mathrm{e}}$ siècle, d'expositions de panneaux peints avec " présentation et portraiture ", i.e., de portrait et d'armoiries, censés rester durant un an au-dessus de la chapelle seigneuriale. Cité dans M. NASSIET, Représentation funéraire et portrait au tournant des XIV et $\mathrm{XVI}^{\mathrm{e}}$ siècles, Bretagne, art, création et société. En l'honneur de Denise Delouche, éd. J.Y. ANDrIeux, M. GrIVEL, Rennes, 1997, p. 91-95.

28. Sur ces représentations du roi René, le corps du prince, figuré en transi, a perdu tous ses traits à proprement parler indispensables au portrait. Seules ses armoiries le font connaître à Dieu et aux hommes. En ce sens le discours héraldique est bien « portrait » dans le sens médiéval du terme définit par SCHMITT, La mort, les morts et le portrait : un ensemble de traits qui établissent une ressemblance dans une perspective eschatologique. Ce thème iconographique, déjà visible sur le célèbre tombeau du cardinal de La Grange, un des premiers transis connus, se retrouve dans divers portraits votifs associant, sur la face, le portrait du donateur, et, au revers ses armoiries associées à une tête de mort (voir par exemple JAN GossAERT, Diptyque Carondelet, 1517, Paris, Louvre, Département des peintures, Inv. 1442, 1443 ; Rogier VAN Der Weyden, Triptyque de la famille Braque, v. 1450, PARIs, Louvre, Département des peintures, Inv. R.F. 2063). Sur le roi mort, voir R.M. FerRé, Le Roi Mort : une image édifiante de la mort de soi, Splendeurs de l'enluminure. Le roi René et les livres, éd. M.É. Gautier, F. AvriL, Angers, 2009, p. 190-197.

29. Sur cette question de l'ange écuyer voir mon article Aux armes saint Michel! Analyse d'un support emblématique performant : l'ange scutifère, Autour de l'archange Saint-Michel. Actes du colloque international organiséà Saint-Michel d'Aiguilhe et au Puy-en-Velay, les 16-17 octobre 2009, éd. M. DE Framond, C. LauransonRosaz, Le Puy en Velay, 2012, p. 265-278.

30. Voir par exemple J.B. DE VAIVRE, La probable signification politique du changement d'armes des comtes de Bourgogne à la fin du XII ${ }^{e}$ siècle, Recueil du $11^{e}$ Congrès international des sciences généalogique et héraldique, Liège, 29 mai-3 juin 1972, Bruxelles, 1973, p. 499-506 ; ID., Échanges et adoptions d'armoiries au XIII ${ }^{\mathrm{e}}$ siècle, Comptes rendus des séances de l'Académie des Inscriptions et Belles-Lettres, t. 126, 1982, p. 371-383 ; ID., Échanges d'armoiries durant la seconde décennie du XIII e siècle, Archivum heraldicum, t. 95, 1981, p. 15-19. 
la plupart du temps, aucune information ne nous est parvenue pour expliquer ce choix. Par ailleurs, des « légendes héraldiques » postérieures sont fréquemment venues brouiller les origines des armoiries ${ }^{31}$. La recherche en la matière a toutefois pu suggérer quelques pistes d'analyse confirmées par l'historiographie récente. Pour ce qui concerne les premières armoiries adoptées par les grands féodaux notamment, les motifs géométriques et les couleurs retenus semblent parfois liés à des emblèmes préexistants, $\mathrm{d}^{\prime}$ origine carolingienne ${ }^{32}$.

Quand le système se développe, dans la seconde moitié du XII siècle, le poids symbolique ou religieux des figures semble avoir été déterminant dans les critères de choix. Enfin, il apparaît qu'au tournant du XIII ${ }^{e}$ siècle, une part importante des motifs des armes originales est choisie sur le principe des figures «parlantes » en relation avec le nom - patronyme, nom de terre, surnom - de leur titulaire. Ces influences multiples n'empêchent pas qu'un choix soit nécessairement posé par l'utilisateur de ces signes mais sa latitude d'action dans la composition de cet outil de représentation reste très difficile à établir. Cette capacité d'intervention des utilisateurs ne semble pas nécessairement s'accroître à mesure que l'héraldique se diffuse dans la société. En effet, les armes des seigneurs et des chevaliers du XIII ${ }^{\mathrm{e}}$ siècle dérivent fréquemment du prototype initial des armes du suzerain. Malgré l'introduction de quelques variantes distinctives, elles constituent alors un ensemble homogène que l'on qualifie de "groupe héraldique ». Au sein même du lignage, les armoiries deviennent rapidement héréditaires - limitant de fait l'intervention personnelle de leur titulaire dans leur composition -, l'aîné conservant des armes strictement identiques à celles du père - les armes dites pleines - et les cadets introduisant des variantes qui ne doivent pas occulter le prototype, les brisures. Les femmes quant à elles portent presque exclusivement les armes pleines de leur père et, après leur union, celles associées de leur père et de leur époux.

L'extension des armoiries à l'ensemble de la société ne remet pas radicalement en cause ces modes de composition, la fonction «parlante » ou les principes de transmission notamment, même si, l'enjeu politique et social n'étant pas le même que dans la noblesse féodale, la latitude de choix a pu être plus ample. Si l'armoirie constitue bien un portrait emblématique, on ne peut pourtant pas, dans la plupart des cas, parler pour leur composition d'autoportrait.

Cette assertion mérite toutefois d'être nuancée au vu des pratiques héraldiques qui se développent dès la fin du XIV ${ }^{e}$ siècle, sans doute dans le but de

31. Cette question des légendes héraldiques n'a pour l'instant jamais fait l'objet d'une synthèse, mais plusieurs travaux universitaires sont en cours.

32. Pour une approche générale du problème, voir Les origines des armoiries. II colloque international d'héraldique, Bressanone-Brixen, 5-9.X.1981, éd. H. Pinoteau, M. Pastoureau, M. Popoff, Paris, 1983. Cette question est actuellement reprise dans les travaux de J.F. NiEus. Voir son article à paraître Pourquoi les armoiries ? Culture chevaleresque et construction identitaire de la haute aristocratie au XII ${ }^{\mathrm{e}}$ siècle. 
pallier en partie cette dimension a-personnelle des armoiries héritées. Nous reviendrons plus loin sur les signes placés autour de l'écu qui permettent de singulariser des armoiries familiales. Mais la période développe également une autre pratique qui aboutit à ce même résultat et que l'héraldique qualifie de "combinaisons d'armoiries ». Cet usage consiste à rassembler sur un même écu les armes des différents fiefs dont on est titulaire, héritier putatif ou présomptif ou encore celles de ses ascendants les plus prestigieux. Cette pratique se retrouve également, à la fin du XIII ${ }^{\mathrm{e}}$ siècle, dans les assemblages d'armoiries résultant d'alliances matrimoniales qui figurent sur un seul écu les armes de l'époux et celles du père de l'épouse. Elle produit donc en un temps donné un signe pour une personne donnée ${ }^{33}$. En effet, à moins d'homonymie héraldique, seule une unique femme pourra porter les armes associées de son mari et de son père, ni sa mère ni sa fille n'utiliseront les mêmes signes héraldiques. Dans le contexte politique, cette pratique se développe à la fin du Moyen Âge, notamment chez les ducs de Bourgogne de la maison de Valois qui « augmentent » tour à tour leurs armes de celles des terres dont ils héritent, la Bourgogne pour Philippe le Hardi (écartelée avec ses armes de cadet de la maison de Valois), la Flandre pour Jean sans Peur (ajoutée en écusson sur ce même écartelé), le Limbourg et le Brabant pour Philippe le Bon (ajoutés en $6^{\mathrm{e}}$ et $7^{\mathrm{e}}$ quartiers). Charles le Téméraire se distingue, avant son avènement, par un lambel (brisure habituellement portée par les aînés). Cette "mode » des combinaisons connaît une sorte d'apogée dans l'héraldique de la maison d'Anjou, en particulier avec les armoiries du roi René composées au plus de sept quartiers ${ }^{34}$. À ce catalogue politique héraldique, sa seconde épouse Jeanne de Laval associe ses propres armes à cinq quartiers qui font état quant à elles de sa généalogie (Laval, Évreux, Navarre, Vitré). Ce type de combinaisons produit, à partir d'armoiries partagées par le lignage, des associations qui ne peuvent appartenir qu'à un seul, en un moment donné, et dont l'organisation hiérarchique même est le résultat d'un choix du titulaire ${ }^{35}$. Véritable tableau généalogique et manifeste politique, ces armes composées sont bien un autoportrait de leur titulaire $^{36}$. Ce type d'armoiries connaît un grand succès, à partir de la fin du

33. Ce qui permet à l'historien de dater très exactement certaines armoiries qui ne peuvent avoir existé que pour une personne précise à un moment précis. Sur cette pratique et sa relation à l'identité, voir M. NAssiet, Alliance et filiation dans l'héraldique des $\mathrm{XIV}^{\mathrm{e}}$ et $\mathrm{XV}^{\mathrm{e}}$ siècles, Revue française d'Héraldique et de Sigillographie, t. 64, 1994, p. 9-29.

34. Entre 1443 et 1453 , René d'Anjou porte des armes à sept quartiers (Hongrie, Naples, Jérusalem, Anjou, Bar, Lorraine, Aragon). Sur ces démonstrations héraldiques, voir C. DE MÉRINDol, Le Roi René et la seconde maison d'Anjou, emblématique, artet histoire, Paris, 1987 ;L. HАвLот, Mise en signe du livre, mise en scène du pouvoir. Armoiries et devises dans les manuscrits de René d'Anjou, et mes notices Lettres d'anoblissement et de concession d'armoiries, Splendeurs de l'enluminure, p. 167-177, 253-257, not. 15-17 ; ID., L'emblématique du roi René, outil de pouvoir et de gouvernement, René d'Anjou (1409-1480). Pouvoirs et gouvernement, éd. J.M. Matz, N.Y. TonNERRE, Rennes, 2011, p. 327-337.

35. Dans l'écu, toutes les positions ne se valent pas. Le coin supérieur gauche (en chef, à dextre pour le porteur de l'écu) est l'espace le plus honorable. René d'Anjou organise par exemple ses quartiers en donnant la place d'honneur aux trois royaumes auxquels il prétend (Hongrie, Naples, Jérusalem) sur les duchés (Anjou, Bar, lorraine), Aragon sur le tout, et organise ces possessions selon l'ordre hiérarchique cité.

36. Pourtant, la diffusion de cette pratique conduit parfois l'ensemble du lignage à adopter la combinaison comme dans le cas des frères et sœur de Jeanne de Laval, l'épouse du roi René. 
$\mathrm{XV}^{\mathrm{e}}$ siècle, dans l'Ouest de la France et dans d'autres régions ${ }^{37}$. Par ailleurs, à la fin de la période dans plusieurs pays d'Europe, méridionale en particulier, notamment l'Italie, les structures du blason s'assouplissent (dès le XIII ${ }^{\mathrm{e}}$ siècle pour la Péninsule ibérique) et les compositions héraldiques intègrent, en plus des armes héritées, des armoiries concédées, voire des emblèmes, qui contribuent également à individualiser le signe héraldique ${ }^{38}$.

Ce souhait essentiel d'entretenir un lien direct, de sujet à sujet, avec le signe chargé de représenter et cette volonté d'agir sur sa composition est régulièrement frustré par la dimension héréditaire du système qui privilégie le groupe à l'individu. Il est invariablement renouvelé dans ce que M. Pastoureau qualifie de « soupapes » de l'héraldique ${ }^{39}$, ces multiples formules qui se développent en marge des armoiries dès la fin du XII ${ }^{\mathrm{e}}$ siècle, à commencer par le cimier.

\section{Le cimier, un autre visage?}

Parmi les signes emblématiques produits par la société médiévale, le cimier $^{40}$, ce décor qui surmonte le heaume du combattant, est assurément un de ceux qui sont le plus directement liés au portrait. En effet, ne couvre-t-il pas la tête, siège de l'âme et de la pensée, et le visage, support de la singularité ? Réalisé en deux ou trois dimensions, sans aucune contrainte formelle ni graphique, le cimier est apparu à la fin du XII $^{\mathrm{e}}$ siècle pour compléter le message identitaire de l'écu armorié, soit par redondance, en en reprenant les motifs et couleurs, soit en développant un discours d'une autre nature emblématique ou symbolique. Véritable échappatoire de l’héraldique, il permet à son utilisateur de personnaliser et d'enrichir sa représentation emblématique, a fortiori si celui-ci porte des armoiries héritées. Investi d'une dimension totémique, le cimier permet au chevalier de devenir un autre soi à l'exemple de Renaud de Dammartin dissimulé sous son cimier aux fanons de baleine durant la bataille de Bouvines en 1214, un des premiers documentés, qui le pare des vertus de ce monstre des mers ${ }^{41}$. Le cimier, en

37. Voir sur le sujet mon article La mémoire héraldique des Visconti dans la France du xve siècle. Quelques considérations sur les stratégies de combinaisons héraldiques à la fin du Moyen Âge, L'Arme segreta. Araldica e storia dell' arte nel Medioevo (secoli XIII-XV), éd. M. FerRari, Florence, 2015, p. 267-283.

38. Cette pratique se développe notamment en Italie au $\mathrm{XV}^{\mathrm{e}}$ siècle.

39. Voir en dernier lieu L'Art héraldique au Moyen Âge, p. 213 s.

40. Sur le cimier, question qui attend encore une synthèse d'envergure, voir notamment Le Cimier. Mythologie, rituel, parenté, des origines au xve siècle. Actes du $6^{e}$ colloque international d'héraldique, La Petite-Pierre, 9-13 octobre 1989, Bruxelles, 1990 ; M. PRINET, Cimiers et supports parlants d'armoiries françaises, Archives héraldiques suisses, 1916, p. 12-21 ; M. Popoff, Le cimier dans la France médiévale. Apparition, diffusion et typologie : l'exemple du Ponthieu, Comunicaciones al xv Congreso internacional de las ciencias genealogica y heraldica, Madrid, 19-25 septiembre 1982, t. 3, Madrid, 1983, p. 357-363.

41. Guillaume Le Breton, La Philippide, trad. M. Guizot, Paris, 1825, chant ix, p. 274, « Son cheval, son bouclier et son casque resplendissant auquel étaient attachées des lames de baleine formant comme deux aigrettes furent vus et reconnus par le roi et par toute l'armée lorsque déjà vainqueurs tous rentraient dans leur camp à la suite de la bataille. ", chant XI, p. 333, "Sur le haut de sa tête le brillant cimier de son casque 
dépit de sa tendance à devenir un marqueur héréditaire dans les panoplies héraldiques de la haute noblesse ${ }^{42}$, garde longtemps et souvent un rapport étroit avec l'individu qui le porte. Sa fonction même - orner le casque qui dissimule et protège la tête - en fait un véritable substitut du visage, un masque à part entière, soit pour devenir quelqu'un d'autre, soit pour sublimer ce que $\mathrm{l}^{\prime}$ on est ${ }^{43}$. Les artistes du Moyen Âge sauront jouer avec talent de cette dimension du signe, d'autant que la plupart de ces cimiers ne sont que des représentations virtuelles imaginées sur le parchemin ou la cire sans devenir jamais des réalités matérielles. Tout est donc permis et la fertile imagination médiévale ne se privera pas d'inventivité dans ce registre comme en témoignent les divers armoriaux qui recensent ces décors de casques ${ }^{44}$. Cette dimension virtuelle ne dévalorise pas la portée emblématique du signe, loin s'en faut. D'abord indissociables des représentations armoriales, les cimiers deviennent bientôt des emblèmes autonomes que l'on retrouve sur certains sceaux - notamment les signets, plus personnels que les sceaux de fonction - dans les marges des manuscrits, sur des monnaies, des frontons de demeures, des graffitis commémoratifs. L'aboutissement de ce processus de représentation semble bien être leur utilisation comme signature dans les documents officiels ou dans le cadre d'échanges d'identités chevaleresques ${ }^{45}$. À côté de cet usage spécifiquement personnel du cimier, se dessine toutefois, dans la haute noblesse et les maisons princières, une nette tendance à l'uniformisation de ce système emblématique qui devient une sorte de totem clanique commun supplantant les divisions généalogiques ou politiques du lignage ${ }^{46}$. En témoignent par exemple les cimiers des cadets de la maison royale. Alors qu'au tournant du xIve siècle les branches capétiennes s'individualisent par des cimiers propres, un siècle plus tard, les «princes des lis » pallient la nature dépréciative des brisures de leurs armes aux fleurs de lis en adoptant tous le même cimier au lis en relief du roi de France ${ }^{47}$.

agite dans les airs une double aigrette, tirée des noires côtes que porte au-dessous de l'antre de sa gueule, la baleine habitante de la mer de Bretagne ; en sorte que le chevalier, déjà grand de sa personne, ajoutant ainsi à sa taille ce bizarre ornement, semblait encore plus grand .»

42. Voir les travaux de J. MorseL, La noblesse dans la mort. Sociogenèse funéraire du groupe nobiliaire

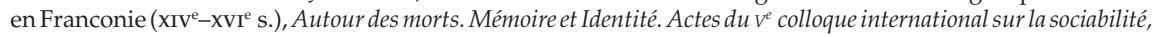
Rouen, 19-21 novembre 1998, éd. O. Dumoulin, F. Thelamon, Rouen, 2001, p. 387-408.

43. M. Pastoureau, Du masque au totem. Le cimier héraldique et la mythologie de la parenté, Razo. Cahier du Centre d'Études médiévales de Nice, t. 6, 1986, p. 39-63, 9 fig.

44. C'est par exemple le cas du Zürcher Wappenrolle (ZüRICH, Schweizerisches Nationalmuseum, cote) ; de l'Armorial du héraut Gelre (Bruxelles, Bibliothèque royale de Belgique, ms. 15652-56).

45. Voir les exemples cités par FrAENKEL, La Signature, p. 260-278. Voir aussi mon article à paraître Masque de guerre et don des armes. Les échanges de cimiers, une pratique chevaleresque à la fin du Moyen Âge, Armes et jeux militaires dans l'imaginaire médiéval (XI-XVe siècles), éd. C. GIRBEA.

46. C'est par exemple le cas du cimier au cygne ou de celui au dragon. Voir Le Cimier ; L. Hablot, Emblématique et mythologie médiévale : le cygne, une devise princière, Animalia, Histoire de l'Art, t. 49, 2001, p. 51-64.

47. Voir mon article Caput regis, corpus regni: le heaume de parement royal à la fin du Moyen Âge, Une histoire pour un royaume (XII-XVe siècle), éd. A.É. Allirot, M. GAude-Ferragu, G. Lecuppre, É. Lequain, L. SCORdiA, J. VÉRONÈSE, Paris, 2010, p. 17-28. 
Armoiries et cimier peuvent donc faire office de portrait à part entière. Ils n'en demeurent pas moins des signes d'individuation autant que d'identification dans la mesure où ils traduisent l'identité dans un cadre graphique et sémiologique commun à un ordre social et porteur d'un sens symbolique - celui de l'identité chevaleresque notamment -, dans la mesure également où l'identité de l'individu y est la plupart du temps absorbée par le groupe du lignage ${ }^{48}$. D'autres systèmes para-héraldiques se développent donc à la fin du Moyen Âge pour tenter de faire exister l'individu et dresser de lui un portrait d'une autre nature, non plus féodale ou lignagère mais morale. $\mathrm{Ce}$ nouveau système de signe est alors désigné sous le nom de devise.

\section{La devise, un autre moi ?}

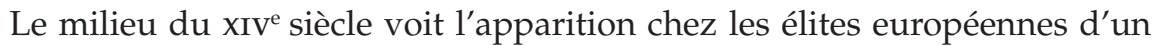
nouveau type d'emblème, les devises. Ces signes para-héraldiques semblent être l'aboutissement des tentatives successives d'expression de l'individualité dans ce registre sémiologique. Délaissant les règles de composition strictes du blason, ces devises adoptent au contraire le trait réaliste qui caractérise la production artistique du temps. Choisies sans aucune restriction de forme ou de sujet, elles peuvent être des animaux, réels ou imaginaires, des plantes - pour lesquelles la fin du Moyen Âge marque un intérêt renouvelé - des objets de toute nature : outil, jeux, armes, vêtements, etc. Un certain nombre de ces signes proviennent ou font office de supports héraldiques, ces figures imaginées dans la première moitié $d u$ XIV siècle pour soutenir l'écu sur les représentations armoriales. Ces devises sont fréquemment associées à une courte sentence qui peut parfois, mais pas nécessairement, préciser le sens de l'emblème. Les sources nomment ces phrases le mot. Celui-ci témoigne de l'intégration de l'écrit dans l'expression emblématique et des influences diverses dont ces nouvelles formules sont issues, notamment les symboles de l'amour courtois et de la foi ${ }^{49}$. Lorsque le système de la devise se diffuse à l'ensemble des élites européennes, il n'est pas rare que ses utilisateurs ne retiennent qu'un unique mot, sans emblème associé. Le terme devise devient alors générique et son sens glisse progressivement pour désigner d'abord les sentences plutôt que les emblèmes. Aux côtés de ces devises et de ces mots, les contemporains s'identifient également au moyen de couleurs symboliques, le plus souvent différentes de celles de leurs armoiries, et de monogrammes ou plus exactement de lettres associées qui ne renvoient pas nécessairement aux initiales de leur prénom mais à une forme élidée du mot

48. J. Morsel, La construction sociale des identités dans l'aristocratie franconienne aux XIV et $\mathrm{XV}^{\mathrm{e}}$ siècles : individuation ou identification ?, L'Individu au Moyen Âge, p. 79-99.

49. Un des registres de signes à l'origine des devises se trouve dans les drueries, ces signes de l'amour courtois que s'échangeaient les amants. Souvent matérialisés en forme de petites broches ou d'anneaux, ces drueries associaient un signe symbolique, animal, végétal, objet, et une courte sentence. Les broches de pèlerinage adoptaient souvent un aspect analogue. 
ou à un énoncé codé. À titre d'exemple et parmi plusieurs milliers de cas, citons l'emblématique de Jean, duc de Berry († 1416), qui porte pour devises l'ours, le cygne blessé, la branche d'oranger, pour mot LE TEMPS VENRA, pour couleurs le rouge, blanc et bleu et pour lettres $\mathrm{E}$ V.

Cette emblématique polymorphe puise donc dans différents registres sémiologiques avec l'évidente ambition de produire le reflet le plus complet possible de l'individu. Sur le plan emblématique d'abord, où il pourra être reconnu à la fois par la devise, le mot, les couleurs ou les lettres, sur le plan symbolique ensuite, où chacun de ces signes portera un sens complémentaire qui définira l'« image » dans laquelle se projette leur utilisateur. Contrairement aux armoiries héritées, parfois même "subies », ces devises sont en effet librement choisies. À la différence des signes héraldiques, qui sont des emblèmes avant d'être des symboles, ces devises sont d'emblée chargées de cette double fonction de signe de reconnaissance (emblème) et d'expression d'une idée, d'un sentiment, d'un projet (symbole). À l'égal des signes héraldiques, en revanche, ces devises existent elles aussi à la place de l'individu et le figurent là où il est absent. La devise est donc bien un portrait emblématique de son titulaire.

Le fait même que le choix de ces signes incombe à leur utilisateur suffit à en faire, dans le principe, un véritable autoportrait. La fréquente association de ces devises, mots, lettres et couleurs avec le portrait physique confirme bien cette lecture ${ }^{50}$. Le premier autoportrait d'artiste reconnu, l'Homme au turban rouge de Jan van Eyck (1433), associe précisément les traits physiques $\mathrm{du}$ peintre à son nom et à son mot $A L S I C H C A N^{51}$. La mise en scène même de ces figures, à plus forte raison quand il s'agit d'animaux que l'on peut couronner, vêtir, parer d'un collier d'ord re de chevalerie, faire trôner, insiste souvent sur ce dédoublement totémique. C'est le cas par exemple de la devise de Bernabo Visconti, co-seigneur de Milan au milieu du XIV siècle : un guépard dans les flammes coiffé du heaume à la guivre des Visconti et tenant son mot SOUFFRIR MESTUET dans un phylactère. Allégorie de la stoïcité (il est l'animal le plus rapide de la terre et pourtant il ne fuit pas et s'assied même dans les flammes) qui est une vertu que prétend incarner Bernabo, cet animal-emblème du prince, quasi comme un totem, porte ici, anthropomorphisé, le propre casque de guerre et de tournoi du prince et semble énoncer lui-même son mot. Cet usage est prolongé dans la littérature politique qui désigne couramment le prince par son emblème, pour l'honorer ou le blâmer.

50. Dans ce registre on peut citer les exemples du Diptyque Wilton de Richard II(LoNDREs, National Gallery, Inv. NG4451), de la bague de Jean sans Peur (PARIs, Louvre, Département des objets d'art, Inv. OA 9524), du portrait de Francesco d'Este (New York, Metropolitan Museum, voir n. 9), celui de Ludovic le More attribué à Giovanni Antonio Boltraffio (Milan, Collection privée). Il faudrait encore compter ici toutes les médailles réalisées pour les princes du $\mathrm{xv}^{\mathrm{e}}$ siècle qui associent sur les deux faces le portrait physique aux devises.

51. Belting, Le portrait médiéval et le portrait autonome, p. 133. L'autoportrait de Jan Van Eyck ne se dispense pas en revanche d'informations d'un autre ordre : le nom, la date, l'emblème. Cet exemple exceptionnel d'autoreprésentation adapte à un quidam - certes artiste reconnu de son vivant comme « le prince des peintres »- les principes d'autoreprésentation des princes du temps. 
Dans le détail, les choses sont toutefois moins claires. En effet, l'importance de ces signes en matière de communication et de représentation justifie probablement un choix concerté du prince et de son conseil. Il ne s'agit plus alors précisément d'un autoportrait. Par ailleurs nous sommes rarement renseignés sur la lecture symbolique qu'il convient de faire de ces signes. Ils sont le plus souvent polysémiques et l'on se garde bien alors de fermer leur lecture par une explicitation officielle pour conserver à l'emblème toute sa capacité de fascination symbolique et tout son potentiel politique. Ce mystère, caractéristique de l'esprit du temps, n'est pourtant pas incompatible avec l'idée de portrait qui n'a pas nécessairement pour objet de tout dire de l'individu qu'il représente. Enfin, la devise n'échappe pas plus que les autres systèmes de signes à cette appropriation du groupe et du lignage. D'outil de représentation personnelle, elle devient souvent signe familial ou dynastique.

Préparée par les tentatives individualisantes de l'expression para-héraldique, notamment le cimier, la devise marque précisément une ultime étape dans laquelle le support, le signifiant, appartient encore en partie aux stéréotypes d'individuation sociale - la culture de cour - tandis que le signifié s'attache déjà à traduire la singularité de la réalité sensible, du visible, du particulier.

\section{La représentation emblématique, autoportrait ou autoreprésentation?}

Les questions qui se posent naturellement quand on cherche à définir la capacité de l'emblématique médiévale à produire un autoportrait sont donc, d'une part, celle de la possibilité pour l'emblème de produire un portrait à proprement parler, et, d'autre part, celle de l'existence d'une relation directe entre l'utilisateur et son portrait emblématique autorisant à parler $\mathrm{d}^{\prime} \ll$ autoportrait ».

Il est évident que ces signes emblématiques s'inscrivent dans un cadre sémiotique précis, lui-même chargé de codes de représentation symboliques et de fonctions socio-juridiques. Se représenter dans un écu soumis aux règles du blason, sur un sceau, via un cimier, ou même à travers une devise, impose le filtre du moyen qui renvoie d'abord à une identité collective, celle du statut ou de la condition : la chevalerie, l'autorité féodale, la société de cour, etc. Cette dimension sociale de l'identité est sans aucun doute longtemps primordiale dans la société médiévale dans laquellel'altérité ou l'individualité peuvent être perçues comme des valeurs négatives - d'autant plus que l'homme médiéval, quels que soient ses traits et son statut, est d'abord et avant tout image de Dieu et que toute production de son image ne prend sens que par rapport à ce «plan divin ». L'image de soi s'inscrit donc dans un modèle d'expression stéréotypé qui définit l'appartenance à un groupe 
et a pour finalité la présence - mémorielle puis physique - au Salut. Le portrait physique lui-même quand il apparaît, n'échappe pas à ce poids du modèle en imposant à la personne représentée tout un ensemble de signes qui le positionnent dans sa société, à commencer par le fait même de se faire représenter. Ce portrait n'échappe pas non plus au «plan divin » dans la mesure où il produit une image inscrite dans le temps, celui du moment de sa réalisation et celui de la mémoire ${ }^{52}$. Mais le portrait physique place en revanche la priorité de ses critères d'identification sur la dissemblance accidentelle propre à chaque être, constituant de fait un « portrait » au sens actuel du terme.

Résoudre ces questions relatives à l'autoportrait emblématique impose donc de les inscrire dans un contexte précis dans lequel il sera possible de cerner l'identité de l'individu sujet, les motivations de la production du portrait, l'action que le commanditaire exerce sur la production de celui-ci, etc. Le portrait princier au tournant $\mathrm{du} \mathrm{xv}^{\mathrm{e}}$ siècle semble un bon cadre $\mathrm{d}^{\prime}$ analyse en raison de la propension que ce groupe socio-politique a eu à se faire représenter sous cette forme, du rôle moteur qu'il a joué en ce domaine, de l'importance de ce type d'image et de l'emblématique en matière de communication, du souci spécifique qui anime les princes dans l'économie du Salut.

Un document tout à fait exceptionnel éclaire notre sujet et fournit un exemple concret de l'interprétation possible des portraits emblématiques, il s'agit de l'anneau dit « de Jean sans Peur », duc de Bourgogne entre 1405 et 1419, conservé aujourd'hui au musée du Louvre ${ }^{53}$. De petite dimension, ce bijou semble avoir été retrouvé sur le corps du prince lui-même lors de la profanation de sa dépouille à la Révolution. Un article de compte laisse par ailleurs penser que cet objet a pu être un présent de son gendre Louis de Guyenne ${ }^{54}$. Cette bague-portrait est une création singulière mais pas unique $^{55}$. Le chaton de cet anneau d'or est composé d'un portrait du profil droit en buste du duc, « de camahieu », une agate sculptée, agrémentée de gemmes qui composent son vêtement-une émeraude - et son chaperon - un jais - ornés tous deux de rubis. Le portrait est réalisé emprès le vif. Il adopte un stéréotype que l'on retrouve sur d'autres représentations du prince,

52. Cette dimension est particulièrement mise en œuvre dans le tableau de Van Eyck, Tymotheos (LoNDRES, National Gallery, Inv. NG290). Une devise y met en scène ce paramètre temps : LEAL SOUVENIR. Sur ce portrait voir SCHMITT, La mort, les morts et le portrait, p. 27-29 ; N. ScHNEIDER, Contribution à l'art du portrait chez Jan van Eyck, Le Portrait individuel, p. 191-192.

53. Voir n. 50. Sur sa réalisation, voir É. Taburet-Delahaye, L'orfèvre Jean Nicolas et deux clients princiers : Marie de France et Louis de Guyenne, Revue du Louvre. La Revue des Musées de France, t. 5, 2001, p. 29-34 ;É. GondRet-Lebailly, Le Dauphin Louis, duc de Guyenne, et les arts précieux (1409-1415), Bulletin Monumental, t. 163-164, 2005, p. 357-374.

54. Jehan Nicolas, orfevre, pour un annel d'or d'un camahieu, d'un saphir et d'une esmeraude a la semblance de mons. de Bourgogne, donnéa mons. de Bourgogne le jour des estrennes 1411 (Extraits des comptes de l'hôtel du dauphin Louis, duc de Guyenne, PARIs, BnF, ms. fr. 32511, fol. 7v, art. 21, cité par Gondret-Lebailly, Le Dauphin Louis, duc de Guyenne, et les arts précieux.

55. Notons toutefois que nous en connaissons d'autres exemples, un réalisé pour ce même duc, d'autres pour son oncle Jean de Berry. Catalogue (É. Taburet-Delahaye, Not. Anneau de Jean sans Peur, Les Princes des fleurs de lis. L'Art à la cour de Bourgogne. Le mécénat de Philippe le Hardi et de Jean sans Peur (1364-1419), Paris, 2004, p. 134, not. 55). 
notamment le folio $126 \mathrm{du}$ Livre des Merveilles ${ }^{56}$. L'ensemble reprend les couleurs emblématiques du duc de Bourgogne : vert, blanc, noir. Une inscription en volume et émaillée, aujourd'hui illisible, entourait l'anneau, peut-être son mot ICH HALTZ MICH (je me tais). À l'intérieur, sous le chaton, se retrouve la devise du rabot, rehaussée d'émail, ainsi que la sentence évangélique partiellement lisible : Vere (filius Dei erat) iste (Matthieu 27, 54). La devise du rabot a été choisie par le duc en 1405. Elle met en signe son projet politique $\mathrm{du}$ Bon gouvernement qui a pour ambition $\mathrm{d}^{\prime}$ « aplanir » les obstacles - à commencer pas son cousin Louis d'Orléans - et de réformer le royaume ${ }^{57}$. La sentence évangélique, incroyablement présomptueuse dans ce contexte, est plus délicate à interpréter. Il peut s'agir d'un clin d'œil du donateur. Notons toutefois que cette citation s'expose à l'intérieur de l'anneau et reste donc invisible. Évoquant la mort du Christ sur la croix et sa reconnaissance post mortem par le Centurion, elle renvoie à la commémoration du défunt et à la réception du message salvifique. S'il est légitimement, à l'égal de tout homme, " fils de Dieu », Jean sans Peur est aussi alors engagé dans un conflit politique majeur pour lequel il peut se considérer comme le promoteur d'une vérité nécessaire à la diffusion de la Vérité.

Destiné à être porté par le prince en une sorte de dédoublement de soi, cet objet en dit long sur la conception médiévale du portrait et sa relation à l'emblématique. Sorte de synthèse de signes qui renvoient tous à une seule et même réalité : le duc de Bourgogne, ces éléments le disent en chair, en signes figurés, en couleurs et en lettres. Tous sont une part de ce qu'il est aux yeux de ses contemporains et surtout, à ses propres yeux. Inscrit dans le temps - celui de l'âge du prince et celui des emblèmes qui varient au fil des circonstances -, cet autoportrait emblématique établit l'image de ce que le prince veut être hic et nunc en vue de son salut. Porté au doigt de ce même seigneur, en contact direct avec sa chair, exposé en permanence à ses yeux, cet anneau constitue une sorte de projection dans laquelle Jean sans Peur se contemple avec empathie, en un constant rappel de l'image qu'il propose, de son projet politique, de sa vocation princière. Ce procédé d'auto-contemplation, de projection empathique, aujourd'hui bien connu est couramment utilisé dans l'iconographie religieuse et la mystique médiévale ${ }^{58}$. Appliqué à la vie politique, il permet sans doute au prince de transcender son être, ce qui est, semble-t-il, le principe même de l'autoportrait.

Université de Poitiers

(CESCM - UMR 7302)
Laurent HABLOT laurent.hablot@univ-poitiers.fr

56. F. AvrIL, Le Livre des Merveilles, manuscrit français 2810 de la bibliothèque nationale de France, Marco Polo, Le Livre des merveilles, éd. M.H. Tesnière, M.T. Gousset, F. Avril, Paris, 1999, p. 197-221, spéc. p. 198, Portrait de Jean sans Peur.

57. L. Навцот, L'emblématique de Philippe le Hardi et de Jean sans Peur, Les Princes des fleurs de lis, p. 81-83 ; ID., La devise, un nouvel emblème pour les princes du $\mathrm{xv}^{\mathrm{e}}$ siècle, La création artistique en France autour de 1400, p. 177-192.

58. Voir par exemple l'article d'E. KöNIG, La réalité du portrait dans les manuscrits enluminés, Le Portrait individuel, p. 167-189. 